\title{
Evaporation of ethanol-water binary mixture sessile liquid marbles
}

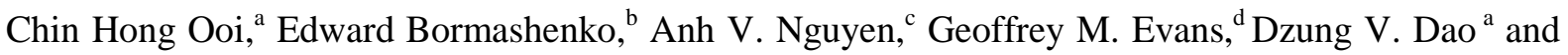
Nam-Trung Nguyen $*^{a}$

a Queensland Micro- and Nanotechnology Centre, Griffith University, 170 Kessels Road, 4111 Queensland, Australia. E-mail: nam-trung.nguyen@griffith.edu.au

${ }^{\mathrm{b}}$ Chemical Engineering and Biotechnology Department, Ariel University, P.O.B. 3, 40700 Ariel, Israel

c School of Chemical Engineering, University of Queensland, St Lucia, 4072 Queensland, Australia.

d School of Engineering, University of Newcastle, Callaghan, NSW 2308, Australia

\begin{abstract}
Recently, numerous works have been reported on the evaporation of a sessile liquid marble, a liquid droplet coated with particles, through geometric measurements. The lack of gravimetric measurement limits further understanding of the physical changes of a liquid marble during the evaporation process. Moreover, the evaporation process of a marble containing a liquid binary mixture has not been reported. This paper studies the effective density and effective surface tension of an evaporating liquid marble which contains aqueous ethanol at relatively low concentrations. The effective density of an evaporating liquid marble is determined from the instantaneous mass and volume measurements. Subsequently, density measurements combined with surface profile fitting provides the instantaneous effective surface tension of the marble. We found that the density and surface tension of the evaporating marble is greatly affected by the particle coating.
\end{abstract}

\section{Introduction}

A liquid marble is a liquid droplet coated with hydrophobic powder. The powder covering the liquid droplet forms a porous coating ${ }^{1,2,3}$ that prevents direct contact between the liquid content and the substrate on which the marble is resting. It is well known that this coating can even allow a liquid marble to float on a liquid surface ${ }^{4,5,6,7,8,9,10}$. The porous coating also allows for both gas exchange 
and evaporation to take place ${ }^{11,12,13,14,15}$. For monolayer coatings the evaporation rate can actually be higher than that for an uncoated droplet ${ }^{16}$. In most cases, however, a multi-layered coating is formed which has a lower evaporation rate ${ }^{9,17,18,19,19}$. Some applications take advantage of the lower evaporation rate of the multi-layered coating to extend the lifetime of the liquid marble ${ }^{11,20}$. Whilst this is a desirable property, the controlled manufacture of multi-layered coatings is challenging given it involves a combination of natural aggregation and random arrangement of the microscopic powder. Increased evaporation rates can also be beneficial for formation of shells made of bound granules ${ }^{21}$. Evaporation of a droplet resting on a hydrophobic powder bed can even cause the powder to coat the droplet spontaneously ${ }^{22}$. The quantitative relationship between evaporation rate of a liquid marble and the coating structure is not fully understood, and for this reason there have been a number of recent studies on the subject ${ }^{9,17,18,19}$.

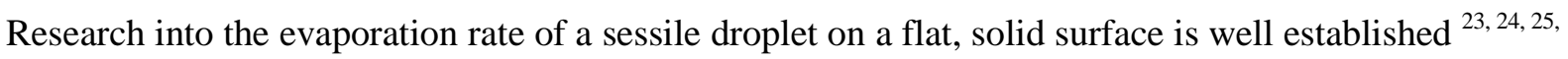
26, 27, 28, 29, 30, 31, 32 , and is comprehensively reviewed by Erbil ${ }^{33}$. The evaporation rate has usually been quantified by monitoring the change in contact angle, volume, mass or shape over time. Evaporation can occur under a constant contact angle or a constant contact area mode ${ }^{34}$, depending on the liquid and surface type. Evaporation mode has also been reported to change with time giving rise to distinct evaporation phases $28,29,31,32,35,36$. Similar studies have been conducted for binary mixture droplets ${ }^{35}$, $36,37,38,39,40$, where discrepancy has been reported with some theories governing evaporation of pure liquid droplets. A similar disparity exists for pure liquid marbles ${ }^{18}$. To the authors’ knowledge only one study exists (Bhosale, Panchagnula and Stretz ${ }^{41}$ ) that has investigated evaporation from a binarycomponent liquid marble; and that was for the water-poly(diallyldimethylammonium chloride) (PDDA) system where only the water was evaporated since the PDDA is not volatile.

Recent interest in autonomously moving and floating liquid marbles based on the Marangoni solutocapillary effect ${ }^{42,43,44}$ has also highlighted the need for better understanding of the evaporation rates and surface tension around a binary-phase liquid marble. For this reason, this study is aimed a quantifying evaporation behaviour and effective surface tension of a binary water-ethanol liquid marble. In particular, we investigated the relationship between effective surface tension, density and 
Bond number. Finally, the evaporation process up to the point of buckling only was considered, as we have yet to develop a proper method for measuring the properties of an irregularly-shaped liquid marble. The instantaneous concentration of the binary mixture is still undetermined as our measurement methods treat the binary liquid content and the coating powder as a single entity.

\subsection{Geometric analysis}

The evaporation rate can be deduced by analysing the geometry of an evaporating droplet. The side view of the droplet is subject to image analysis to measure its contact angle and contact radius. Droplet volume can then be calculated based on these measurements. For isothermal, diffusioncontrolled evaporation of a sessile pure droplet, Erbil, McHale and Newton developed the following expression for the droplet volume as a function of the constant contact angle ${ }^{34}$ :

$V_{c}^{2 / 3}=V_{c i}^{2 / 3}-\frac{2}{3} K\left(M_{w}, T, P_{v s}, D, R H, \rho, \theta\right) f(\theta) t$

where $V_{c}$ is the instantaneous volume of the droplet. $V_{c i}$ is the initial volume of the droplet. $K$ is a constant which is assumed to be independent of time and volume and can be determined from the parameters listed in the adjacent parentheses. $M_{w}$ is the molecular weight of the liquid, $T$ is the temperature, $P_{v s}$ is the saturated vapour pressure, $D$ is the diffusion coefficient, $\rho$ is the density, $R H$ is the relative humidity of the droplet environment, $\theta$ is the contact angle and $t$ is time. The full expression of $K$ and $f$ can be found in the literature ${ }^{17,19,33,34,45}$. Erbil, McHale and Newton ${ }^{34}$ also developed an expression for the contracting contact radius as a function of time assuming a constant contact angle. Equation (1) provides a useful prediction of the evaporation rate if the droplet and environmental properties are known at all times. This is possible if the usage of pure liquids on surfaces with low imperfections are used. For the case of a binary mixture however, $K$ will vary with time since saturated vapour pressure, diffusion coefficient, density and contact angle are timedependent. The time functions for each of these parameters is needed to calculate $K$.

For a droplet consisting of water mixed with a more volatile component, its contact radius decreases

over time whilst its contact angle usually increases at first and then decreases eventually $35,37,38,39,40$. 
The reduction in contact radius is expected as the droplet volume and surface area decreases over time. The contact angle depends on the surface tension of the droplet in accordance with Young's contact angle equilibrium. Surface tension is a function of the concentration of the binary mixture, and contact angle changes have been reported ${ }^{35,37}$ as a result of evaporation of the different components. The instantaneous concentration of the binary mixture droplet can be deduced from the droplet profile or the contact angle, but is subjected to the effects of surface imperfections. Trunk, Popp and Kiefer were able to directly measure instantaneous concentration of levitated droplets using Raman spectroscopy ${ }^{46}$, which allowed them to calculate the evaporation rates of the individual components.

Wang et al. ${ }^{39}$ related contact angle, $\theta$, and contact radius, $R$, for the final evaporation phase using the following expression:

$\theta=a_{1} R^{3}+a_{2} R$

where $a_{1}$ and $a_{2}$ denote fitting coefficients that will be influenced by intrinsic properties of the droplet such as ethanol concentration. In fitting Equation (2) to the experimental results contact radius is assumed to be linearly proportional to time, which is yet to be tested for the case of a liquid marble.

Studies involving liquid marbles report that measurement of the contact angle is very difficult due to the presence of the "fluffy" coating ${ }^{17}$. Moreover, where the contact angle was able to be measured, it was found not always constant ${ }^{17,18}$. To the best of the authors' knowledge, there are only a few studies that reported the contact angles and contact radii of an evaporating liquid marble. Tosun and Erbil ${ }^{18}$ and Doganci et al. ${ }^{19}$ reported an evaporating marble that had a decreasing contact angle similar to that of a bare droplet. Conversely, Doganci et al. ${ }^{19}$ reported an evaporating marble that had an increasing contact radius, which is the opposite behaviour of a bare droplet. Possibly, this was the result of sagging of the marble during the evaporation process.

The evaporation resistance model of La Mer ${ }^{47}$ for a liquid droplet has been used to compare the evaporation resistance parameter, $\phi$, of a liquid marble and a liquid droplet ${ }^{17,18,19}$ : La mer's model is given by: 
$\phi=A_{c}\left(\frac{M_{w} P_{v s}}{R_{g} T}\right)(1-R H)\left(\frac{d t}{d m_{m}}-\frac{d t}{d m_{l}}\right)$

where $A_{c}$ is the area of the evaporating droplet with an assumed spherical cap shape, $R_{g}$ is the gas constant, $\mathrm{d} t / \mathrm{d} m_{m}$ and $\mathrm{d} t / \mathrm{d} m_{l}$ are the reciprocals of the rate of change of mass for the liquid marble (assuming monolayer coating) and liquid droplet, respectively. Equation (3) is based on the assumption of a monolayer coating, which is not the case for liquid marbles ${ }^{1,8,9,18}$ unless customized methods and materials are used ${ }^{48}$. Also, it is assumed in the volume calculation that the liquid marble maintains a constant axisymmetrical shape - like that of a liquid droplet - which is no longer valid once the marble coating collapses (buckles) inwards. It is therefore unlikely that Equation (3) will be satisfactory when quantifying evaporation rates from liquid marbles, especially when buckling of the coating layer takes place.

\subsection{Gravimetric analysis}

Evaporation rates can be measured by monitoring the mass of the droplet. Bhosale, Panchagnula and Stretz established a linear relationship between surface area and time at a given evaporation phase of the liquid marble ${ }^{41}$. The surface area was calculated from the accurately measured mass and known density, with the assumption that the liquid marble formed a perfect sphere. Four distinct evaporation phases were observed, with the final phase attributed to the non-volatility of the dissolved PDDA. The mass of the liquid marble was measured using a thermogravimetric analyzer under a highly controlled environment. The study reported change in surface area of the evaporating marble as a function of time. Neither contact angle or contact radius was reported due to the difficulty in their measurement. Shi et al. ${ }^{40}$ conducted both gravimetric and geometric analysis on an evaporating ethanol-water droplet on PTFE. The instantaneous mass, contact angle and contact radius were reported for the pure liquids and the binary mixture. The marble volume was not reported. Contact line pinning and depinning was observed during the evaporation process. 


\section{Materials and Methods}

\subsection{Liquid marble preparation and characterisation}

Ethanol (Chem-Supply 99.5\% purity) was diluted with distilled water to obtain the desired concentrations. The aqueous ethanol droplet was then dispensed onto a powder bed of loose polytetrafluoroethylene (PTFE) powder (Sigma-Aldrich ${ }^{\circledR} 1 \mu \mathrm{m}$ nominal diameter, $\rho=2.2 \mathrm{~g} / \mathrm{cm}^{3}$ ). For accurate volume control, a micropipette (Thermo Scientific Finnpipette 4500 0.5-10 $\mu \mathrm{l}$ ) was used as the dispenser. The micropipette had an uncertainty of $\pm 4.3 \%$. The liquid marble was formed by rolling the aqueous ethanol droplet in the powder bed, followed by rolling it around on a clean stainless steel spoon to dislodge excess powder from the marble surface. The liquid marble was then placed on an electronic balance (RadWag® AS82/220.R2 Analytical Balance).

The mass of the marble coating, $m_{c}$ was found by subtracting the mass of the liquid marble, $m_{\mathrm{m}}$, with the mass of the liquid content, $m_{l}$, i.e. $m_{c}=m_{m}-m_{l}$. The mass of liquid $m_{l}$ was calculated as $m_{l}=\rho_{l} V_{l}$, where $\rho_{1}$ and $V_{l}$ are liquid density and volume, respectively. The value of $\rho_{1}$ was obtained from literature ${ }^{49}$, whilst the value of $V_{\mathrm{l}}$ was controlled by the micropipette. Both $m_{\mathrm{m}}$ and $m_{\mathrm{l}}$ were measured 10 times each. A $10 \mu \mathrm{l}$ liquid droplet dispensed by the micropipette weighed $10.08 \pm 0.36 \mathrm{mg}$. The mean mass of a $10 \mu 1$ liquid marble was $10.21 \mathrm{mg}$, which was well within the uncertainty range of the micropipette.

The methodology used was incapable of measuring the coating powder mass of an individual liquid marble. For this reason, the mass of the coating was determined by averaging the coating powder of 20 marbles. Firstly, the mass of an empty container was measured. Twenty liquid marbles with a volume of $10 \mu \mathrm{l}$ each were then placed into the container, and the mass of the container with liquid marbles was measured and then left to dry for 2 days. With the liquid completely removed from the container, only the powder remained; and the difference in mass of the container with the powder and the blank container yielded the mass of the coating of 20 liquid marbles. Five readings were taken for each measurement to determine the data uncertainty. The average mass of the coating powder for one liquid marble was found to be $0.25 \pm 0.05 \mathrm{mg}$. The average water mass removed from the drying 
process was $10.07 \pm 0.03 \mathrm{mg}$ per liquid marble, which is almost the same as the mass of a $10 \mu \mathrm{l}$ droplet dispensed by the micropipette. Measurement results are shown in Table 1.

Table 1 Raw data of container and mass of 20 liquid marbles. All values shown in mg.

\begin{tabular}{lrrr}
\cline { 2 - 4 } & $\begin{array}{c}\text { Container } \\
\text { only }\end{array}$ & $\begin{array}{c}\text { Container } \\
\text { with marbles }\end{array}$ & $\begin{array}{c}\text { Container with } \\
\text { powder }\end{array}$ \\
\cline { 2 - 4 } & 10479.09 & 10685.04 & 10483.14 \\
& 10478.81 & 10685.18 & 10483.46 \\
& 10478.62 & 10685.77 & 10483.75 \\
& 10479.06 & 10685.82 & 10484.87 \\
& 10479.16 & 10685.36 & 10484.70 \\
\hline Mean & 10478.95 & 10685.43 & 10483.98 \\
Standard deviation & 0.23 & 0.35 & 0.76
\end{tabular}

\subsection{Liquid marble gravimetric measurements}

All measurements were conducted in a laboratory environment at a temperature of $293.5 \pm 0.5 \mathrm{~K}, 1$ atmospheric pressure and a relative humidity of $57.3 \pm 3 \%$. Electronic balance sensitivity drift was characterized without the auto-zero feature. The resulting correction factor was used to reduce systematic errors from the mass measurements. The electronic balance was also calibrated before each run. The mass of the liquid marble was logged by the electronic balance at a fixed time interval of 180 s. The corresponding side views of the evaporating marble were acquired by a horizontally-mounted USB camera (EO ${ }^{\circledR}-5012 C$ 1/2" CMOS Color USB Camera with $1.0 \mathrm{X}$ telecentric lens). The camera focus was adjusted using a linear stage motor (Zaber Technologies T-LS28M). Figure 1 shows the experimental setup.

\subsection{Liquid marble geometric measurements}

The side views of the liquid marble were analyzed using ImageJ (National Institutes of Health, USA) with the low-Bond axisymmetric drop shape analysis ${ }^{50}$ (LB-ADSA) plugin. This method allowed rapid, accurate and non-destructive measurement of the geometry of the drop. The initial generated drop profile was manually placed in the vicinity of the marble on screen and fitted using gradient energy optimization ${ }^{50}$. The drop profile is dependent on curvature, density and surface tension. Using the plugin, the drop profile was repeatedly optimized until it matched that of the real droplet. 
Geometric parameters of the droplet were then obtained by solving the Young-Laplace equation for the fitted droplet profile. The parameters generated by the software include the apparent contact angle $\theta$, apex curvature $b$, inverse square of capillary length $c$, contact area $A$, marble volume $V_{\mathrm{m}}$, and surface area $A_{s}$. From the known drop volume and mass, the effective density was calculated using $\rho_{\mathrm{m}}=m_{\mathrm{m}} / V_{\mathrm{m}}$. Given that the inverse square of capillary length is $c=\rho_{\mathrm{m}} g / \gamma_{\text {eff }}$, where $\gamma_{\text {eff }}$ is the effective liquid marble surface tension, and $g$ is the gravitational acceleration of $9.81 \mathrm{~m} / \mathrm{s}^{2}$, and that $c$ and $\rho_{\mathrm{m}}$ are known, then the effective surface tension $\gamma_{\text {eff }}$ was able to be calculated. Note that the effective liquid marble surface tension $\gamma_{\text {eff }}$ is different from that of the liquid-air $(\gamma)$ or solid-air $\left(\gamma_{\mathrm{SA}}\right)$ surface tensions because the surface is neither purely liquid nor solid. It is actually a mixture of liquid and embedded solid powder.

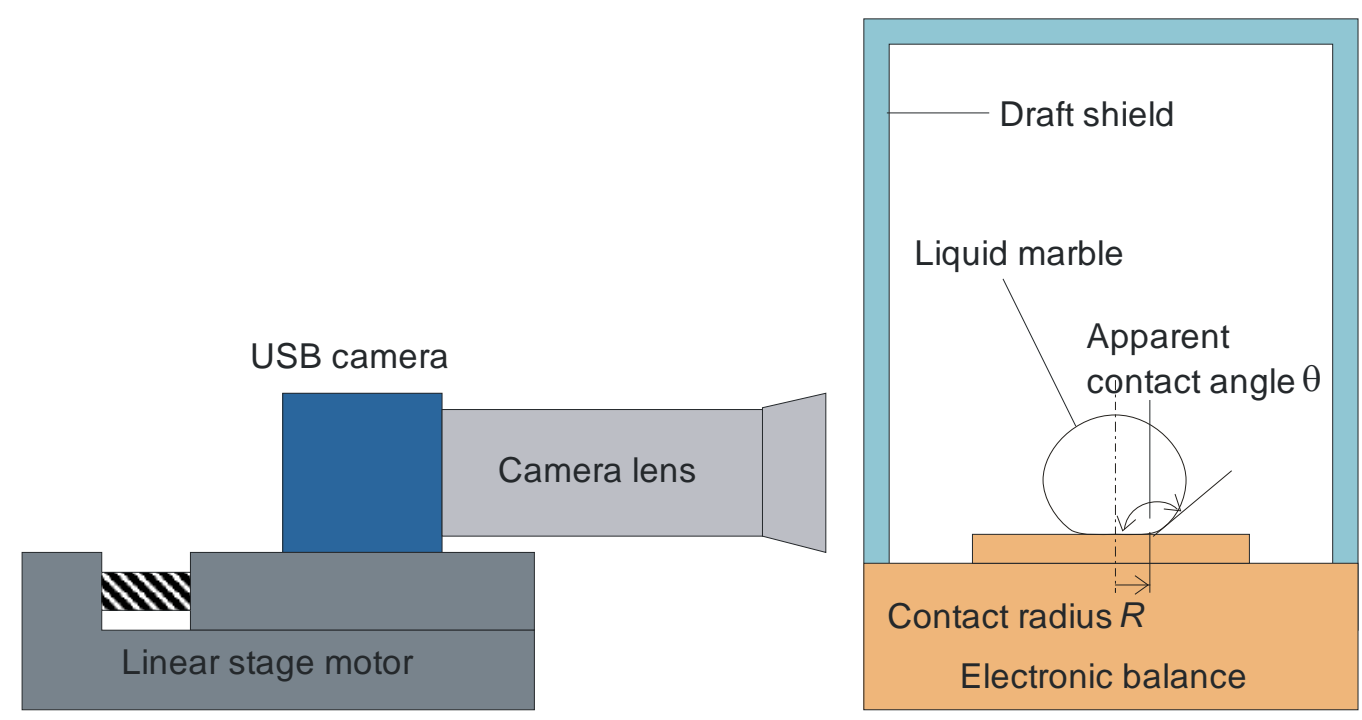

Fig 1 Experimental setup. Liquid marble and the PTFE plate were placed on weighing pan of electronic balance fitted with draft shield which was not airtight to allow air exchange and prevent vapour saturation within the weighing chamber.

For liquid marble experiments, data were collected until the onset of buckling, beyond which the model assumption of an axisymmetric shape was no longer satisfied. 


\section{Results and discussion}

Figure 2 compares the normalized mass measurements of aqueous ethanol droplets of different ethanol volume concentrations. Normalized mass was obtained by dividing the instantaneous mass with the initial mass, $m^{*}=m / m_{0}$. As expected, droplets with higher ethanol concentration have steeper curves due to the higher evaporation rates. Pure water and pure ethanol curves serve as the two limiting cases of the measurements. The aqueous ethanol droplets have higher evaporation rates initially, but then gradually reduce to a level similar to that of pure water. Our results are similar to those of Shi et al. ${ }^{40}$. For aqueous ethanol droplets at higher ethanol concentrations, volume estimates were not computed because the droplets did not form axisymmetrical shapes. The high wettability due to the presence of ethanol caused the droplets to form irregular shapes which render visual-based volume measurements highly inaccurate. This problem was not as apparent for the aqueous ethanol marble as the particle coating prevented direct physical contact between the aqueous ethanol and the weighing pan surface. 


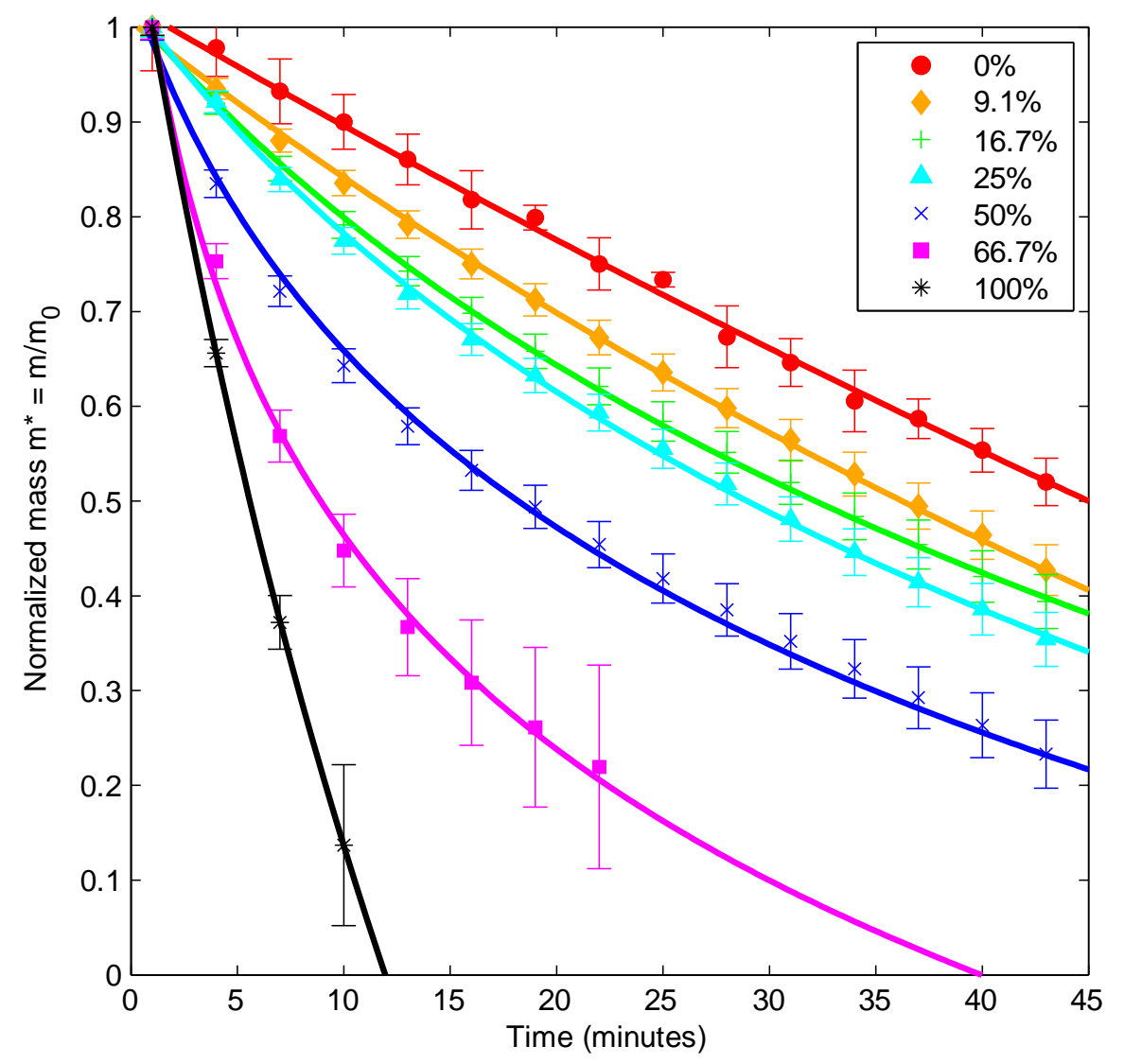

Fig 2 Normalized mass of aqueous ethanol droplets with different ethanol volume concentrations. Fitting lines are shown for reference. Error bars indicate measurement uncertainties. All measurements were conducted with an initial droplet volume of $10 \mu \mathrm{l}$.

Figure 3 shows the volume, mass, contact angle and contact radius measurements for aqueous ethanol marbles. The normalized volume $V^{*}=V / V_{0}$ and normalized mass are shown in Figure 3(a) and 3(b), respectively. For ethanol concentrations less than $25 \%$ the evaporation trends were similar but less in magnitude to that of the uncoated droplet. The lower evaporation rate of a marble can be attributed to (1) a direct reduction in the available mass transfer area (due to the presence of particles on the droplet surface); and (2) the presence of air, ethanol and water vapours trapped within the porous multi-layered coating. Figure 3(c) and 3(d) show no particular trend for either the marble contact angle or contact radius; noting that the reported contact angles are apparent angles as the fluffy appearance obscured the actual edges. This result is unlike that for either pure water marbles or binary mixture droplets as shown in previous studies ${ }^{35,38,39}$. Evaporating binary mixture droplets have 
decreasing contact angles and radii. This is consistent with ethanol volume concentration as low as $12.3 \%{ }^{39}$. Decreasing contact angle has also been observed for an evaporating pure water marble ${ }^{18}$. Measurements with ethanol concentrations higher than 25\% were attempted, but the marbles were very unstable and ruptured upon placement. For ethanol volume concentrations at $66.7 \%$ or higher, the droplet simply wetted and seeped through the PTFE powder bed without a marble being formed. This phenomenon has been reported previously ${ }^{51}$.

At low ethanol concentrations ( $<25 \%)$, the evaporation rate can be linearly approximated for both the liquid droplet and marble. The slope of these lines are compared as shown in Table 2. Liquid marbles have lower evaporation rates at ethanol concentrations up to $25 \%$, which is consistent with that of a pure water marble where the evaporation rate is lower than that of a pure water droplet ${ }^{17,18}$.

Table 2 Comparison of evaporation rates between a liquid droplet and liquid marble at different ethanol concentrations. Evaporation rate is defined as the slope of the normalized mass versus time plot where time is measured in minutes.

\begin{tabular}{|c|c|c|}
\cline { 2 - 3 } \multicolumn{1}{c|}{} & \multicolumn{2}{c|}{ Evaporation rate m*/t } \\
\hline $\begin{array}{l}\text { Ethanol } \\
\text { concentration (\%) }\end{array}$ & Droplet & Marble \\
\hline 0 & 106 & 104 \\
\hline 9 & 157 & 106 \\
\hline 17 & 190 & 130 \\
\hline 25 & 206 & 145 \\
\hline
\end{tabular}



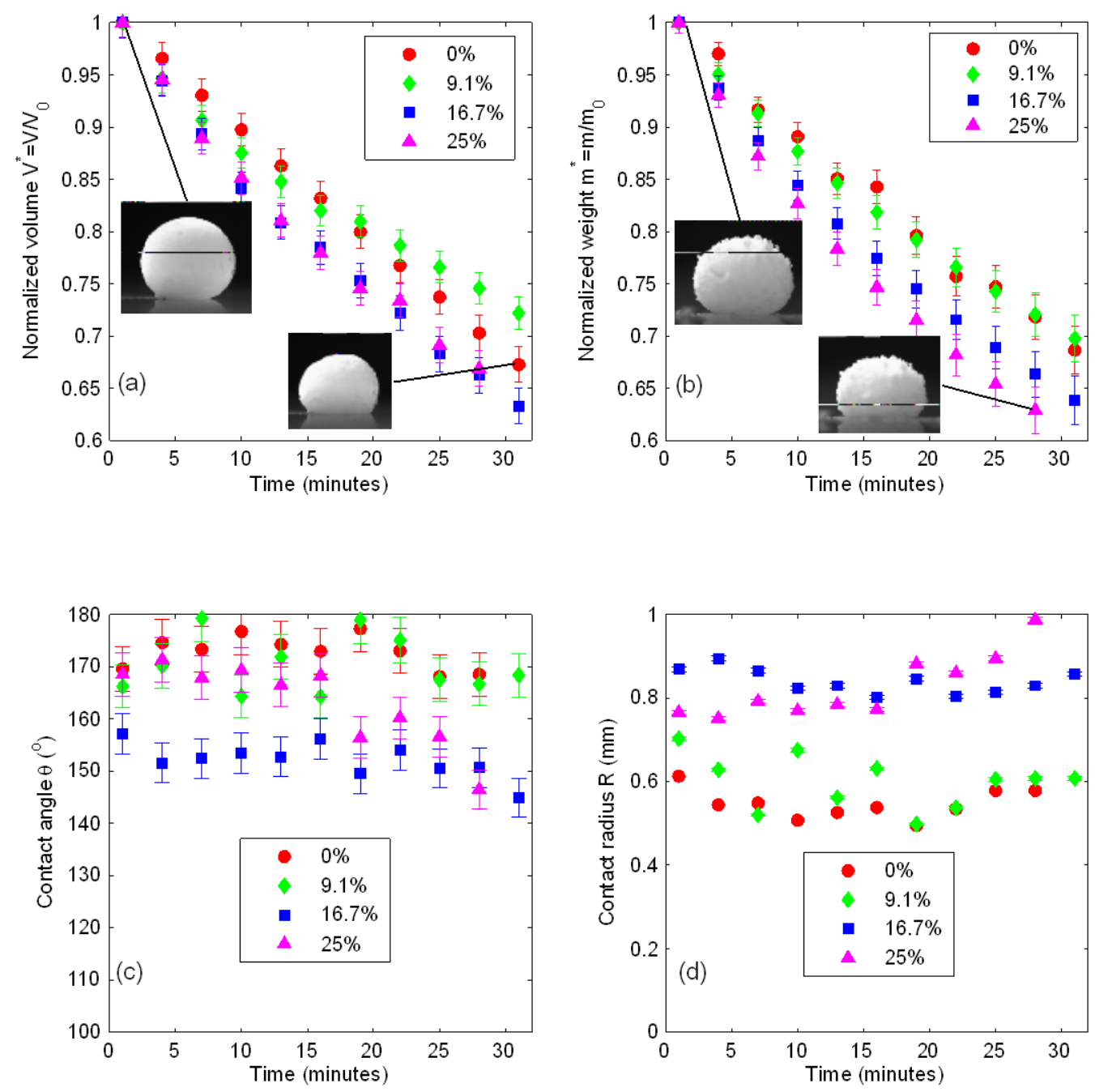

Fig 3 Measured parameters of aqueous ethanol marbles for different ethanol volume concentrations. (a) Normalized volume. (b) Normalized mass. (c) Apparent contact angle. (d) Contact radius. Photo insets show examples of side view of pure water and aqueous ethanol marbles. All runs were conducted with an initial marble volume of $10 \mu \mathrm{l}$.

Figure 4(a) shows overlapping droplet profiles plotted as a function of time. The contact line of the droplet was first pinned for about 18 minutes, with the droplet evaporating at constant contact radius mode. Then, the right side of the droplet de-pinned and the droplet proceeded to evaporate at a constant contact angle mode. The left side of the droplet was pinned throughout the measurementsmost probably due to surface imperfections. The trends observed in Figure 4(a) are consistent with other studies that found that an evaporating sessile liquid droplet on a solid surface initially evaporates 
with constant contact radius which is then proceeded by evaporation under constant contact angle mode ${ }^{52,53}$. The corresponding overlapping liquid marble profiles plotted as a function of time are shown in Figure 4(b). For a liquid marble containing the same concentration, the contact line might not be pinned to the solid surface since the marble is non-wetting. Consequently, the marble evaporated with a monotonically decreasing contact radius. However, it can be seen that the contact radius stopped decreasing after some time, and since the contact line was not pinned then the top of the marble began collapsing.
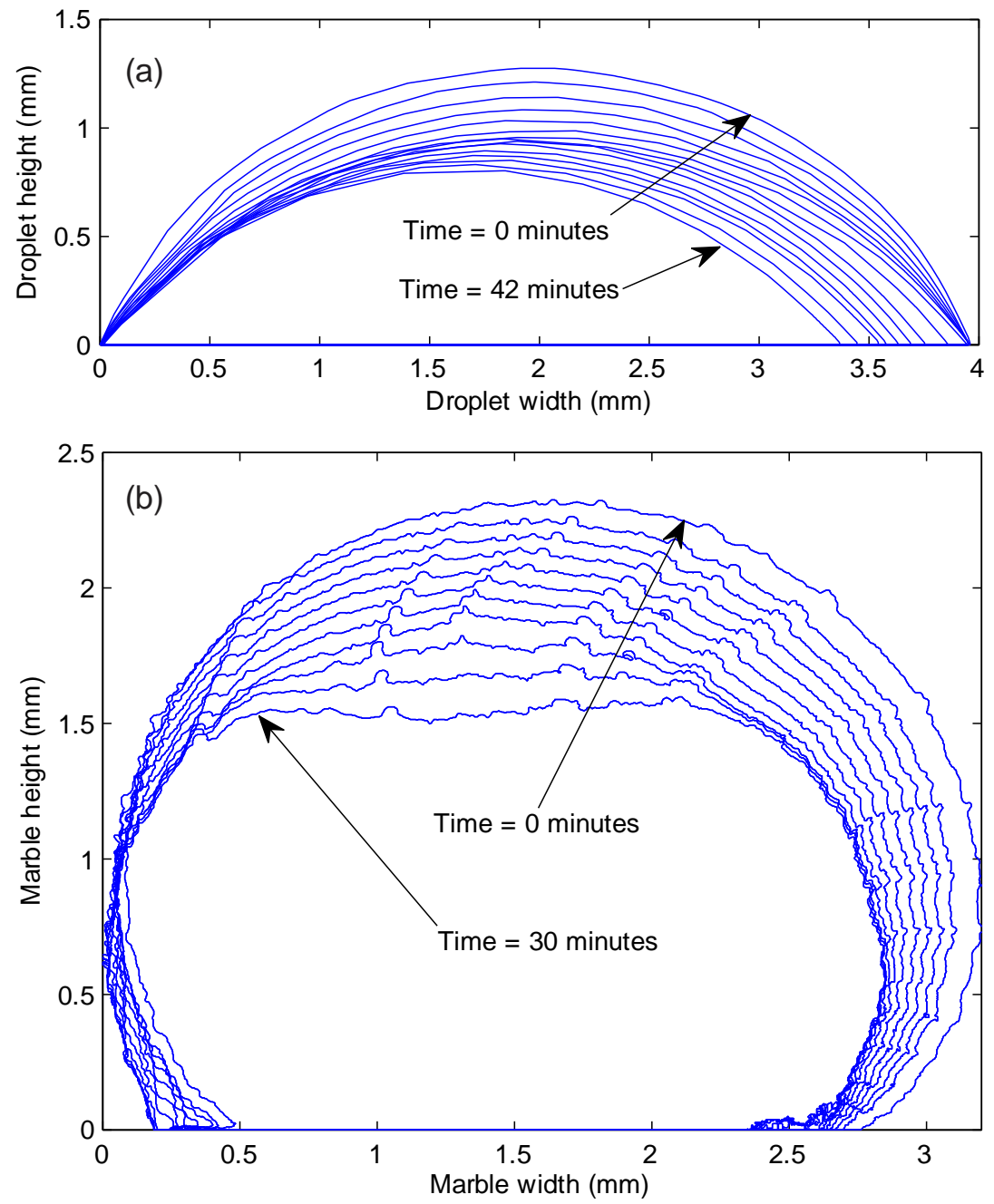

Fig 4 Outlines of an evaporating (a) droplet and (b) marble containing $10 \mu \mathrm{l}$ aqueous ethanol at 16.7\% concentration. Each outline is spaced 3 minutes apart, starting from the outermost line. The jagged edges in (b) are caused by the uneven profile of the powder coating. 
Figure 5(a) shows the effective density of the aqueous ethanol marble plotted against time. It can be seen that the effective density either increased or decreased with time. From previous work ${ }^{35}$ the volatile compound in the binary mixture should evaporate first, and it would be expected that the effective density should increase and eventually equal that of pure water once all of the more volatile component had evaporated.
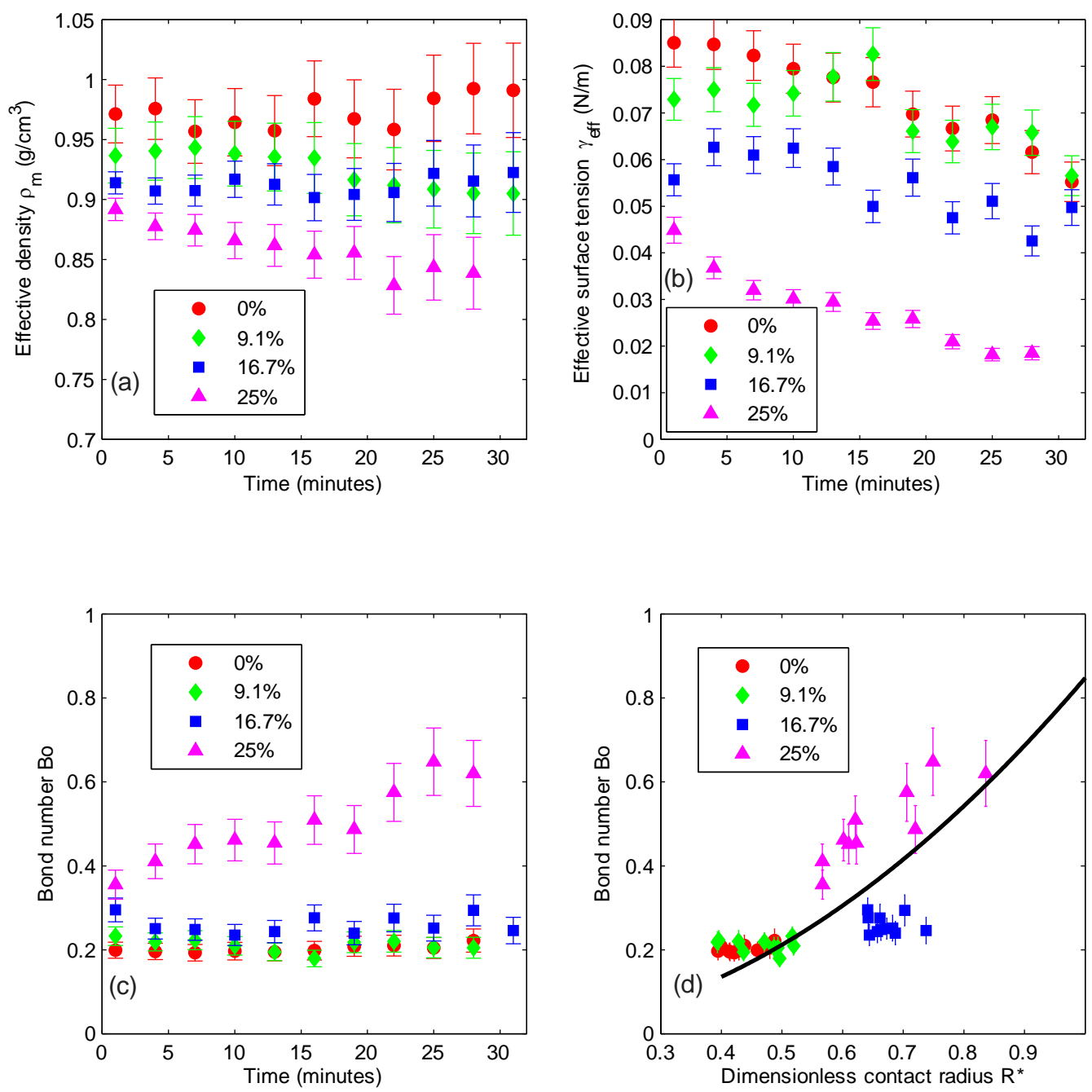

Fig 5 Calculated parameters of the aqueous ethanol marbles with different ethanol volume concentration. (a) Effective density. (b) Effective surface tension. (c) Bond number. (d) Bond number versus dimensionless contact radius. The solid line represents the relationship Bo $=3 / 2 R *^{2}$. 
The observation that for some ethanol concentrations the effective density actually decreased with time could be because the coating layer was occupied by air and/or vapour which would make it less dense than the liquid inside the marble. To test this hypothesis, the mass and volume of the coating layer are required in accordance with the following expression:

$\rho_{\mathrm{c}}=\frac{m_{\mathrm{c}}}{V_{\mathrm{c}}}=\frac{m_{\mathrm{c}}}{V_{\mathrm{m}}-V_{\mathrm{l}}}=\frac{m_{\mathrm{c}}}{V_{\mathrm{m}}-m_{\mathrm{l}} / \rho_{\mathrm{l}}}=\frac{m_{c}}{V_{\mathrm{m}}-\left[m_{\mathrm{m}}-m_{\mathrm{c}}\right] / \rho_{\mathrm{l}}}$

where $\rho$ refers to effective density, $m$ refers to mass and $V$ refers to volume of a liquid marble component depending on the subscript. Subscripts $c$ corresponds to the coating layer which includes trapped air, $m$ refers to the marble and $l$ refers to the liquid content within the marble. From the measured quantities of $V_{\mathrm{m}}, m_{\mathrm{m}}, m_{\mathrm{c}}$ and $\rho_{\mathrm{l}}, \rho_{\mathrm{c}}$ is determined to be $0.635 \mathrm{~g} / \mathrm{cm}^{3}$ on average for a $10 \mu \mathrm{l}$ pure water marble. This is well below the liquid density even at $25 \%$ ethanol concentration $\left(\rho_{l}=0.946\right.$ $\mathrm{g} / \mathrm{cm}^{3}$ ) and could contribute to the reduction in effective density of the marble even though evaporation of the more volatile component is taking place. The volume of trapped air, $V_{\mathrm{a}}$ occupies $4.18 \%$ of the total marble volume on average. The effective density for an ethanol marble cannot be determined using the current methodology because $\rho_{1}$ changes with time as the ethanol evaporates.

Figure 5(b) shows that the effective surface tension, $\gamma_{\text {eff }}$, of the aqueous ethanol marble has decreased against time. As the marble shrinks, the surface area decreases even though the particle coating remains in place. The liquid-air interface that contributes to the marble effective surface tension is gradually replaced with layers of particle coating ${ }^{1}$. From thermodynamic considerations the effective surface tension of a liquid marble can be expressed as:

$\gamma_{\mathrm{eff}}=\frac{\gamma\left(S-S_{0}\right)+\left(\gamma_{\mathrm{SL}}+\gamma_{\mathrm{SA}}\right) S_{0}}{S}=\gamma+\left(\gamma_{\mathrm{SL}}+\gamma_{\mathrm{SA}}-\gamma\right) S_{0} \frac{1}{S}$

where $\gamma_{\mathrm{SL}}, \gamma_{\mathrm{SA}}$ and $\gamma$ are the surface tensions at the powder-liquid, powder-air, and liquid-air interfaces, respectively; $S$ is the total surface of the liquid marble, and $S_{0}$ is the area of the marble occupied by powder. As the liquid content of the marble shrinks so does its volume and surface area. The powder coating shrinks accordingly to conform to the marble surface thereby increasing the ratio 
$S_{0} / S$ with time. In accordance with Equation (5), this will result in a decrease in $\gamma_{\text {eff }}$; but $\gamma_{\text {eff }}$ could not be calculated since $S_{0}, \gamma_{\mathrm{SL}}, \gamma_{\mathrm{SA}}$ were not measured. By extending this explanation, it is likely that the liquid content eventually recedes to a point where it detaches from the powder coating, forming a shell. This powdered shell could be held weakly in place by an evaporating thin water film. As the powder particle amount becomes relatively excessive, the surface tension provided by the water film diminishes and gravity causes the top of the shell to buckle.

Figure 5(c) shows the Bond number, Bo, as a function of time, where Bo is given by:

$$
\mathrm{Bo}=\frac{\rho_{\mathrm{m}} g r_{0}^{2}}{\gamma_{\text {eff }}}
$$

where $r_{0}=(3 V / 4 \pi)^{2 / 3}$ is the radius of the undeformed spherical marble. It can be seen that Bo is relatively constant for ethanol concentrations below $16 \%$. This is attributed to the reduction in marble density being more or less compensated for by corresponding decrease in effective surface tension. At $25 \%$ ethanol concentration, it can be seen that the Bo actually increased with time.

For small sessile marbles with contact radius smaller than its capillary length, the contact radius can be estimated as ${ }^{3,54}$ :

$$
\begin{aligned}
& R=\sqrt{\frac{2 \rho_{\mathrm{m}} g}{3 \gamma_{\mathrm{eff}}}} r_{0}^{2} \\
& \frac{R}{r_{0}}=\sqrt{\frac{2 \rho_{\mathrm{m}} g}{3 \gamma_{\mathrm{eff}}}} r_{0}=\sqrt{\frac{2 \rho_{\mathrm{m}} g r_{0}^{2}}{3 \gamma_{\mathrm{eff}}}}=\sqrt{\frac{2 \mathrm{Bo}}{3}}
\end{aligned}
$$

Rearranging (7) results in the relationship between the Bond number and the dimensionless contact radius $R^{*}=R / r_{0}$ :

$$
\mathrm{Bo}=\frac{3}{2}\left(\frac{R}{r_{0}}\right)^{2}=\frac{3}{2}(R *)^{2},
$$

The solid line in Figure 5(d) depicts the relationship (8), which fits well for a small $R^{*}$ or a small marble volume. 


\section{Conclusion}

An aqueous ethanol marble has similar evaporation trend to an uncoated droplet in terms of volume reduction at low ethanol concentrations. Compared to uncoated droplets, marbles have lower evaporation rates at all attempted ethanol concentrations. Setups for accurate geometric measurements at higher concentration droplets are problematic due to their highly wettable characteristics, which produce low contact angles and asymmetric shapes. On the other hand, high concentration marbles can hardly be formed and are usually extremely fragile.

The results showed that apparent contact angles and contact radii do not show obvious trends, unlike uncoated droplets of binary mixtures. The effective density and effective surface tension of a marble are influenced by the instantaneous ethanol concentration and the particle coating density.

The particle coating density seems to be the major factor for our case. However, equipment with higher sensitivity and a highly controlled environment is needed to accurately characterize the coating particles. The Bond numbers of liquid marbles seem to show different behaviour at higher ethanol concentrations. Future works using more stable liquid marbles at high ethanol concentrations will be needed to confirm this observation. 


\section{References}

1. Bormashenko, E.; Musin, A.; Whyman, G.; Barkay, Z.; Starostin, A.; Valtsifer, V.; Strelnikov, V. Revisiting the surface tension of liquid marbles: Measurement of the effective surface tension of liquid marbles with the pendant marble method. Colloids Surf. A 2013, 425 (0), 15-23.

2. $\quad$ Aussillous, P.; Quere, D. Liquid marbles. Nature 2001, 411 (6840), 924-7.

3. Aussillous, P.; Quere, D. Properties of liquid marbles. Proc. R. Soc. A 2006, 462 (2067), 973999.

4. Zhang, L.; Cha, D.; Wang, P. Remotely Controllable Liquid Marbles. Adv. Mater. 2012, 24 (35), 4756-4760.

5. $\quad$ Gao, L.; McCarthy, T. J. Ionic Liquid Marbles. Langmuir 2007, 23 (21), 10445-10447.

6. Bormashenko, E.; Musin, A. Revealing of water surface pollution with liquid marbles. Appl. Surf. Sci. 2009, 255 (12), 6429-6431.

7. Bormashenko, E.; Bormashenko, Y.; Musin, A. Water rolling and floating upon water: marbles supported by a water/marble interface. J. Colloid Int. Sci. 2009, 333 (1), 419-21.

8. Bormashenko, E.; Bormashenko, Y.; Musin, A.; Barkay, Z. On the mechanism of floating and sliding of liquid marbles. ChemPhysChem 2009, 10 (4), 654-6.

9. Cengiz, U.; Erbil, H. Y. The lifetime of floating liquid marbles: the influence of particle size and effective surface tension. Soft Matter 2013, 9 (37), 8980-8991.

10. Ooi, C. H.; Vadivelu, R. K.; St John, J.; Dao, D. V.; Nguyen, N.-T. Deformation of a floating liquid marble. Soft Matter 2015, 11 (23), 4576-4583.

11. Sarvi, F.; Jain, K.; Arbatan, T.; Verma, P. J.; Hourigan, K.; Thompson, M. C.; Shen, W.; Chan, P. P. Y. Cardiogenesis of Embryonic Stem Cells with Liquid Marble Micro-Bioreactor. Adv. Healthc. Mater. 2014, 77-86.

12. Tian, J.; Arbatan, T.; Li, X.; Shen, W. Liquid marble for gas sensing. Chem. Commun. 2010, 46 (26), 4734-4736.

13. Tian, J.; Arbatan, T.; Li, X.; Shen, W. Porous liquid marble shell offers possibilities for gas detection and gas reactions. Chem. Eng. J. 2010, 165 (1), 347-353.

14. Arbatan, T.; Shen, W. Measurement of the Surface Tension of Liquid Marbles. Langmuir 2011, 27 (21), 12923-12929.

15. Arbatan, T.; Al-Abboodi, A.; Sarvi, F.; Chan, P. P.; Shen, W. Tumor inside a pearl drop. Adv. Healthc. Mater. 2012, 1 (4), 467-9.

16. Laborie, B.; Lachaussee, F.; Lorenceau, E.; Rouyer, F. How coatings with hydrophobic particles may change the drying of water droplets: incompressible surface versus porous media effects. Soft Matter 2013, 9 (19), 4822-4830.

17. Dandan, M.; Erbil, H. Y. Evaporation rate of graphite liquid marbles: comparison with water droplets. Langmuir 2009, 25 (14), 8362-7. 
18. Tosun, A.; Erbil, H. Y. Evaporation rate of PTFE liquid marbles. Appl. Surf. Sci. 2009, 256 (5), 1278-1283.

19. Doganci, M. D.; Sesli, B. U.; Erbil, H. Y.; Binks, B. P.; Salama, I. E. Liquid marbles stabilized by graphite particles from aqueous surfactant solutions. Colloids Surf. A 2011, 384 (1-3), 417-426.

20. Tian, J.; Fu, N.; Chen, X. D.; Shen, W. Respirable liquid marble for the cultivation of microorganisms. Colloids Surf. B 2013, 106 (0), 187-190.

21. Eshtiaghi, N.; Liu, J. J. S.; Hapgood, K. P. Formation of hollow granules from liquid marbles: Small scale experiments. Powder Technology 2010, 197 (3), 184-195.

22. Whitby, C. P.; Bian, X.; Sedev, R. Spontaneous liquid marble formation on packed porous beds. Soft Matter 2012, 8 (44), 11336-11342.

23. Hu, H.; Larson, R. G. Evaporation of a Sessile Droplet on a Substrate. J. Phys. Chem. B 2002, 106 (6), 1334-1344.

24. Picknett, R. G.; Bexon, R. The evaporation of sessile or pendant drops in still air. J. Colloid Int. Sci. 1977, 61 (2), 336-350.

25. Rowan, S. M.; Newton, M. I.; McHale, G. Evaporation of Microdroplets and the Wetting of Solid Surfaces. J. Phys. Chem. 1995, 99 (35), 13268-13271.

26. Deegan, R. D.; Bakajin, O.; Dupont, T. F.; Huber, G.; Nagel, S. R.; Witten, T. A. Contact line deposits in an evaporating drop. Phys. Rev. E Stat. Nonlin. Soft Matter Phys. 2000, 62 (1), 756-765.

27. Poulard, C.; Guéna, G.; Cazabat, A. M. Diffusion-driven evaporation of sessile drops. J. Phys. Condens. Matter 2005, 17 (49), S4213.

28. Dash, S.; Garimella, S. V. Droplet evaporation dynamics on a superhydrophobic surface with negligible hysteresis. Langmuir 2013, 29 (34), 10785-95.

29. Pittoni, P. G.; Chang, C.-C.; Yu, T.-S.; Lin, S.-Y. Evaporation of water drops on polymer surfaces: Pinning, depinning and dynamics of the triple line. Colloids Surf. A 2013, 432, 89-98.

30. Hu, D.; Wu, H.; Liu, Z. Effect of liquid-vapor interface area on the evaporation rate of small sessile droplets. Int. J. Therm. Sci. 2014, 84, 300-308.

31. Kovalchuk, N. M.; Trybala, A.; Starov, V. M. Evaporation of sessile droplets. Curr. Opin. Colloid. Interface Sci. 2014, 19 (4), 336-342.

32. Cheng-Kun, C.; Yen-Wen, L. Evaporation phase change processes of water/methanol mixtures on superhydrophobic nanostructured surfaces. J. Micromech. Microeng. 2011, 21 (7), 075003.

33. Erbil, H. Y. Evaporation of pure liquid sessile and spherical suspended drops: A review. Adv. Colloid Interface Sci. 2012, 170 (1-2), 67-86.

34. Erbil, H. Y.; McHale, G.; Newton, M. I. Drop Evaporation on Solid Surfaces: Constant Contact Angle Mode. Langmuir 2002, 18 (7), 2636-2641.

35. Sefiane, K.; Tadrist, L.; Douglas, M. Experimental study of evaporating water-ethanol mixture sessile drop: influence of concentration. Int. J. Heat Mass Transfer 2003, 46 (23), 4527-4534. 
36. Liu, C.; Bonaccurso, E.; Butt, H.-J. Evaporation of sessile water/ethanol drops in a controlled environment. Phys. Chem. Chem. Phys. 2008, 10 (47), 7150-7157.

37. Sefiane, K.; David, S.; Shanahan, M. E. R. Wetting and Evaporation of Binary Mixture Drops. J. Phys. Chem. B 2008, 112 (36), 11317-11323.

38. Cheng, A. K. H.; Soolaman, D. M.; Yu, H.-Z. Evaporation of Microdroplets of Ethanol-Water Mixtures on Gold Surfaces Modified with Self-Assembled Monolayers. J. Phys. Chem. B 2006, 110 (23), 11267-11271.

39. Wang, Z.; Peng, X.-F.; Mujumdar, A. S.; Su, A.; Lee, D.-J. Evaporation of Ethanol-Water Mixture Drop on Horizontal Substrate. Drying Technol. 2008, 26 (6), 806-810.

40. Shi, L.; Shen, P.; Zhang, D.; Lin, Q.; Jiang, Q. Wetting and evaporation behaviors of waterethanol sessile drops on PTFE surfaces. Surf. Interface Anal. 2009, 41 (12-13), 951-955.

41. Bhosale, P. S.; Panchagnula, M. V. On Synthesizing Solid Polyelectrolyte Microspheres from Evaporating Liquid Marbles. Langmuir 2010, 26 (13), 10745-10749.

42. Bormashenko, E.; Bormashenko, Y.; Grynyov, R.; Aharoni, H.; Whyman, G.; Binks, B. P. Self-Propulsion of Liquid Marbles: Leidenfrost-like Levitation Driven by Marangoni Flow. J. Phys. Chem. C 2015, 119 (18), 9910-9915.

43. Nakata, S.; Nagayama, M.; Kitahata, H.; Suematsu, N. J.; Hasegawa, T. Physicochemical design and analysis of self-propelled objects that are characteristically sensitive to environments. Phys. Chem. Chem. Phys. 2015, 17 (16), 10326-38.

44. Ooi, C. H.; Nguyen, A. V.; Evans, G. M.; Gendelman, O.; Bormashenko, E.; Nguyen, N.-T. A floating self-propelling liquid marble containing aqueous ethanol solutions. RSC Adv. 2015, 5 (122), 101006-101012.

45. Ooi, C. H.; Nguyen, N.-T. Manipulation of liquid marbles. Microfluid Nanofluid 2015, 19 (3), 483-495.

46. Trunk, M.; Popp, J.; Kiefer, W. Investigations of the composition changes of an evaporating, single binary-mixture microdroplet by inelastic and elastic light scattering. Chem. Phys. Lett. 1998, 284 (5-6), 377-381.

47. Mer, V. K. L.; Aylmore, L. A. G.; Healy, T. W. The Ideal Surface Behavior of Mixed Monolayers of Long-chain n-Paraffinic Alcohols. J. Phys. Chem. 1963, 67 (12), 2793-2795.

48. $\quad$ Ogawa, S.; Watanabe, H.; Wang, L.; Jinnai, H.; McCarthy, T. J.; Takahara, A. Liquid Marbles Supported by Monodisperse Poly(methylsilsesquioxane) Particles. Langmuir 2014, 30 (30), 9071-9075.

49. González, B.; Calvar, N.; Gómez, E.; Domínguez, Á. Density, dynamic viscosity, and derived properties of binary mixtures of methanol or ethanol with water, ethyl acetate, and methyl acetate at $\mathrm{T}$ $=(293.15,298.15$, and 303.15) K. J. Chem. Thermodyn. 2007, 39 (12), 1578-1588.

50. Stalder, A. F.; Melchior, T.; Müller, M.; Sage, D.; Blu, T.; Unser, M. Low-bond axisymmetric drop shape analysis for surface tension and contact angle measurements of sessile drops. Colloids Surf. A 2010, 364 (1), 72-81.

51. Whitby, C. P.; Bian, X.; Sedev, R. Rolling, penetration and evaporation of alcohol-water drops on coarse and fine hydrophobic powders. Colloids Surf. A 2013, 436 (0), 639-646. 
52. Stauber, J. M.; Wilson, S. K.; Duffy, B. R.; Sefiane, K. On the lifetimes of evaporating droplets with related initial and receding contact angles. Phys. Fluids 2015, 27 (12), 122101.

53. Nguyen, T. A. H.; Nguyen, A. V. Increased Evaporation Kinetics of Sessile Droplets by Using Nanoparticles. Langmuir 2012, 28 (49), 16725-16728.

54. Mahadevan, L.; Pomeau, Y. Rolling droplets. Phys. Fluids 1999, 11 (9), 2449-2453. 